\title{
Analysis on Parameters of WLF Equation for Viscoelastic Damping Materials
}

\author{
Bing Wang, Guanjun Chang, Xinzhi Lin and Wantao Guo \\ Luoyang Ship Material Research Institute, Luoyang, China
}

\begin{abstract}
Williams-Landel-Ferry equation (WLF equation)is one of the most significant experimental equations in analysis for the temperature frequency dependence of viscoelastic damping materials. The papers about WLF equation are reviewed and the difference of expression for WLF equation is analyzed with emphasis. A new idea, which parameters of WLF equation is related to the temperature scale, is proposed, and the relation for different parameters for WLF equation is also proved.
\end{abstract}

KEYWORD: WLF equation; Viscoelasticity; Master Curve; Temperature Frequency Dependence

\section{INTRODUCTION}

The measured dynamic modulus data curve of amorphous polymers at different temperatures and frequencies can mapped and get a complete modulus curve by reduced frequency, which is discovered by J D Ferry et al (J.D.Ferry, 1952). In fact, storage modulus and the imaginary modulus obey the same moving rule. $\mathrm{R} L \mathrm{~L}$ Williams, J F Landel and J D Ferry(M.L, 1955) found that there is a quantitative relationship between the shift factor and the experimental temperature of the amorphous polymer; that is, the WLF equation named after their name:

$$
\log \alpha_{T}=-C_{1}\left(T-T_{s}\right) /\left[C_{2}+\left(T-T_{s}\right)\right] \text {, }
$$

In eq.(1), $\mathrm{C}_{2}$ and $\mathrm{C}_{1}$ are constants for any given polymer $\mathrm{T}_{0}$ is the reference temperature. This relation reveals the intrinsic link of temperature and frequency, lays the foundation for the expansion the effective measurement frequency range of the materials. J. D. Ferry gives detailed explanation of the WLF equation in the literature (J D Ferry, 1970), and thinks that the parameters of $\mathrm{C}_{1}$ and $\mathrm{C}_{2}$ and the reference temperature $\mathrm{T}_{0}$ change from a kind of polymer to another.

For most of the viscoelastic damping materials, WLF equation reveals certain equivalent relationship which exists between temperature and frequency. WLF equation is an important empirical formula to fit the master curve. In the late seventies of the last century, U. S. air force material testing laboratory according to the equivalent relationship of temperature and frequency for viscoelastic damping materials, the dynamical modulus and loss factor with reduced frequency of $f \bullet \alpha_{T}$ as abscissa, draws out the dynamic mechanical properties of the nomo- gram, also said master curve, has now become the American national standards and ISO standards. And also China also formulates the corresponding national standard GB / T 17809-1999.

A lot of damping material and acoustic literature describes WLF equation, but these differences of the coefficient are not clear. The author analyses the derivation of the WLF equation and a large number of documents, to clarify the origin of different coefficient. The WLF coefficient associates with viscoelastic material, and the reference temperature, and also the relevant temperature scale. While the point has not yet reported in the open literature.

\section{DERIVATION OF WLF EQUATION}

On the basis of the Doolittle equation of liquid viscosity, WLF equation can be derived, the Doolittle equation is shown below:

$\eta=A \exp \left(B V / V_{f}\right)$

In eq.(2), $\eta$ is viscosity. $\mathrm{B}$ and $\mathrm{A}$ are constants; $V$ is total volume; $V_{f}$ is free volume.

When the temperature $\mathrm{T}$ is above glass transition temperature (abbr.Tg), the eq.(2) takes the logarithm, and gets

$\ln \eta(T)=\ln A+\frac{B V(T)}{V_{f}(T)}$

When $\mathrm{T}$ equals $\mathrm{Tg}$,

$$
\ln \eta\left(T_{g}\right)=\ln A+\frac{B V\left(T_{g}\right)}{V_{f}\left(T_{g}\right)}
$$


The Eq.(3) and (4) subtraction, according to the free volume theory can be obtained as

$\ln \frac{\eta(T)}{\eta\left(T_{g}\right)}=B\left[\frac{V(T)}{V_{f}(T)}-\frac{V\left(T_{g}\right)}{V_{f}\left(T_{g}\right)}\right]=B\left[\frac{1}{f_{r}}-\frac{1}{f_{T_{g}}}\right]$

The free volume fraction $f_{r}$ is under the temperature of $\mathrm{T}$ in the high elastic zone.

Free volume fraction $f_{T_{g}}$ is at the glass transition temperature.

$f_{r}$ is defined as $f_{r}=f_{T g}+a_{f}\left(T-T_{g}\right)$, Eq.(5) can be expressed as

$\ln \frac{\eta(T)}{\eta\left(T_{g}\right)}=B\left[\frac{1}{f_{T_{g}}+a_{f}\left(T-T_{g}\right)}-\frac{1}{f_{T_{g}}}\right]$

In eq.(6), $a_{f}$ is free volume expansion of high elastic state.

The eq. (6) is converted to a common logarithmic form as follows,

$\lg \frac{\eta(T)}{\eta\left(T_{g}\right)}=-\frac{B}{2.303 f_{T_{g}}}\left[\frac{\left(T-T_{g}\right)}{f_{T_{g}} / a_{f}+\left(T-T_{g}\right)}\right]=\frac{-C_{1}\left(T-T_{g}\right)}{C_{2}+\left(T-T_{g}\right)}$

Usually, $B$ approximately equal to 1 , $a_{f}=4.8 \times 10^{-4} / \mathrm{K}$

Can be obtained,

$$
\mathrm{C}_{1}=\frac{\mathrm{B}}{2.303 \mathrm{f}_{T g}}=17.44 \mathrm{C}_{2}=f_{T_{g}} / a_{f}=51.6 \mathrm{~K}
$$

Here is $\mathrm{C}_{1}$ and $\mathrm{C}_{2}$ when the reference temperature is the glass transition temperature on the absolute temperature scale. From the derivation process, it can be seen that the scope of application of the formula is above the glass transition temperature. And the temperature range is in the glass transition temperature of more than 100k.

\section{WLF EQUATION COEFFICIENT CONVERSION RELATIONS}

Analysis of the WLF equation expression, it is not difficult to see that $\mathrm{C}_{1}$ is dimensionless, and dimensional unit of $\mathrm{C}_{2}$ and temperature unit should be the same. From this point of view, this argument which the product of $\mathrm{C}_{1}$ and $\mathrm{C}_{2}$ is equal to 900 is not strictly speaking. In many occasions, the reference temperature is not the glass temperature, the new coefficients $\mathrm{C}_{1}$ and $\mathrm{C}_{2}$ can be calculated by using the conversion method. Using the conversion relation, it can be proved the WLF equation coefficient is not only related to the choice of the reference temperature, and also closely related to the temperature scale. With the reference temperature for this view, the literature is also mentioned. However, there is no strict proof about temperature scale connection in the open literature.

For reference temperature $\mathrm{T}_{0}$,

$\log \alpha_{T}=-\frac{C_{1}\left(T-T_{0}\right)}{C_{2}+T-T_{0}}$
When $T_{S}\left(T_{S}=\mathrm{T}_{0}+\delta\right)$ instead of $\mathrm{T}_{0}$ as the reference temperature, Eq.(8) can be expressed as

$\log \alpha_{T}^{\prime}=-\frac{C_{1}^{\prime}\left(T-T_{S}\right)}{C_{2}^{\prime}+T-T_{S}}$

When $\mathrm{T}$ equals $\mathrm{T}_{\mathrm{s}}$, Eq.(8) can be expressed as follow

$\log \alpha_{T_{s}}=-\frac{C_{1}\left(T_{s}-T_{0}\right)}{C_{2}+T_{s}-T_{0}}$

According to the relationship between the polymer relaxation time and the shift factor, then

$\log \alpha_{T}=\log \frac{t}{t_{0}}=-\frac{C_{1}\left(T-T_{0}\right)}{C_{2}+T-T_{0}}$
$\log \alpha_{T_{s}}=\log \frac{t_{s}}{t_{0}}=-\frac{C_{1}\left(T_{s}-T_{0}\right)}{C_{2}+T_{s}-T_{0}}$

In eq.(11) and eq.(12),t is the relaxation time of the polymer at the temperature of T. And $t_{0}$ is the relaxation time of the polymer at the temperature of $\mathrm{T}_{0}$, and $t_{s}$ is the relaxation time of the polymer at the temperature of Ts.

Eq. (11) minus eq. (12) ,as follow,

$\log \frac{t}{t_{s}}=-\frac{C_{1}\left(T-T_{0}\right)}{C_{2}+T-T_{0}}-\left[-\frac{C_{1}\left(T_{s}-T_{0}\right)}{C_{2}+T_{s}-T_{0}}\right]$

According to the relationship between the polymer relaxation time and the shift factor, the eq. (9) can be transformed as

$\log \alpha_{T}^{\prime}=\log \frac{t}{t_{s}}=-\frac{C^{\prime}{ }_{1}\left(T-T_{S}\right)}{C_{2}^{\prime}+T-T_{S}}$

It can be seen that eq. (13) is equal to eq. (14).

$-\frac{C^{\prime}{ }_{1}\left(T-T_{S}\right)}{C_{2}^{\prime}+T-T_{S}}=-\frac{C_{1}\left(T-T_{0}\right)}{C_{2}+T-T_{0}}-\left[-\frac{C_{1}\left(T_{s}-T_{0}\right)}{C_{2}+T_{s}-T_{0}}\right]$

The eq. (15) can be expressed as

$-\frac{C_{1}^{\prime}\left(T-T_{S}\right)}{C^{\prime}{ }_{2}+T-T_{S}}=-\frac{C_{1} C_{2}}{C_{2}+\delta} \cdot \frac{T-T_{0}-\delta}{C_{2}+T-T_{0}}$

It be written as

$\frac{C_{1}^{\prime}\left(T-T_{S}\right)}{C_{2}^{\prime}+T-T_{S}}=\frac{C_{1} C_{2}}{C_{2}+\delta} \cdot \frac{T-\left(T_{0}+\delta\right)}{\left(C_{2}+\delta\right)+T-\left(T_{0}+\delta\right)}$

For $T_{S}$ equals $T_{0}+\delta$, eq. (17) can be written as

$\frac{C_{1}^{\prime}\left(T-T_{S}\right)}{C_{2}^{\prime}+T-T_{S}}=\frac{C_{1} C_{2}}{C_{2}+\delta} \cdot \frac{T-T s}{\left(C_{2}+\delta\right)+T-T s}$

It can be seen from eq. (18),

$C_{1}^{\prime}=\frac{C_{1} \cdot C_{2}}{C_{2}+\delta}$

$C^{\prime}{ }_{2}=C_{2}+\delta$

In general, the reference temperature will be applied in the range of reference temperature up and down about $50 \mathrm{~K}$.

When $\delta$ equals $50 \mathrm{~K}$, according to the eq.(19) and eq.(20), the new parameters of WLF equation can be calculated as

$$
C_{1}=\frac{17.44 \times 51.6}{51.6+50}=8.86 \text {, }
$$


and $\mathrm{C}_{2}=51.6+50=101.6 \mathrm{~K}$. The data is frequently adopted in literature.

The above derivation is based on the absolute (Kelvin) scale of temperature.

The similar conversion calculation of $\mathrm{C}_{1}$ and $\mathrm{C}_{2}$ can be calculated under different temperature scales.

According to the conversion of $\mathrm{T}=273+\mathrm{t}$ between Kelvin and Celsius temperature scales, $C_{1}$ and $C_{2}$ under Kelvin scale the is equal to that under Celsius temperature scale.

And according to the relationship of the conversion of Fahrenheit and the absolute temperature scale, through the derivation of the relationship can be relationship between Fahrenheit and absolute temperature scale of temperature coefficient are as follows:

$$
C_{2}^{\prime}=\frac{9}{5} C_{2}, C_{1}^{\prime}=C_{1}
$$

Through the above analysis, we can see that the WLF equation parameters are affected by the selection of reference temperature and the scale of temperature. A large number of literature results show that the parameters of WLF equation are related to the type of polymer. In this way, there is a large number of various types of WLF equation expression in the literature is not surprising.

The absolute temperature (Kelvin) scale is adopted in ISO4664-1:2005 and GB/T9870.1-2006 standard. DIG Jones formula as the WLF equation can be expressed under the Fahrenheit temperature scale, $\mathrm{C}_{1}=12, \mathrm{C}_{2}=5250 \mathrm{~F}, \mathrm{ASTM}$ have been included in the E755-05 standard. But the same equation is directly adopted in GJB981, which obviously ignore the temperature scale transformation relations. And the author thinks that it is not appropriate.

\section{SUMMARY}

In this paper, the domestic and foreign literatures about various expression forms of WLF equation are summarized. Proposed equation coefficients and the relevant materials, and the reference temperature, especially the coefficient and temperature scale on this view and from the point of view of formula derivation proved equation coefficients and reference temperature and temperature scale on this view, clarify the WLF equation at home and abroad all the intrinsic link between expression.

\section{REFERENCES}

Chang Guanjun, Viscoelastic Damping Materials[M], National Defense Industry Press,2012,156-158

Doolittle A K.Studies in Newtonian flow II.The dependence of the viscosity of liquids on free space[J].J. Appl. Polym.Sci.1976,20(12):3403-3423

He Manjun, Chen Weixiao, Dong Xixia, polymer physics[M]. Fudan University press, 1990,234-235
J.D.Ferry,etc., Temperature dependence of dynamic properties of elastomer:relaxation distributions[J],Industrial and Engineering Chemistry, April,1952,703 706

J D Ferry, Viscoelastic properties of polymers[M], John Wiley \& Sons, 1970

M.L.Williams,R.F.Landel and J.D.Ferry, The temperature dependence of relaxation mechanisms in armorphous polymers and glass-forming liquids[J],J.Amer.Soc., 1955,vol.77, 3701-3707

Zheng Qiang, Lin Yu, "polymer physics" in the teaching of WLF equation coefficient calculation and analysis, polymer bulletin, 2010, sixth, 99-105 